$05,13,01$

\title{
Инжекция неравновесного спина в геликоидальный ферромагнетик
}

\author{
(C) E.A. Караштин \\ ${ }^{1}$ Институт фоизики микроструктур РАН, \\ Нижний Новгород, Россия \\ ${ }^{2}$ Нижегородский государственный университет им. Н.И. Лобачевского, \\ Нижний Новгород, Россия \\ E-mail: eugenk@ipmras.ru
}

Поступила в Редакцию 26 марта 2020 г.

В окончательной редакции 26 марта 2020 г.

Принята к публикации 2 апреля 2020 г.

\begin{abstract}
Исследованы особенности инжекции спина в геликоидальный ферромагнетик. Рассмотрены два способа спиновой инжекции: инжекция спин-поляризованного тока и спиновый пампинг. Для случая, когда ось геликоида перпендикулярна границе, через которую происходит инжекция спина, определены условия, при которых происходит заполнение электронами заданной спиновой подзоны. Для случая, когда ось геликоида параллельна границе раздела, продемонстрировано возникновение эффекта, аналогичного топологическому эффекту Холла. При спиновом пампинге во второй геометрии обнаружено явление конверсии инжектируемого спинового тока в электрический, текущий вдоль оси геликоида, за счет обменного взаимодействия в ферромагнетике.
\end{abstract}

Ключевые слова: неколлинеарный ферромагнетик, спиновый пампинг, топологический эффект Холла, инжекция спина, обменное взаимодействие.

DOI: 10.21883/FTT.2020.09.49773.05H

\section{1. Введение}

Инжекция неравновесного спина в проводящую среду может осуществляться несколькими способами. Один из них заключается в пропускании электрического тока через систему, состоящую из двух ферромагнетиков (проводника, в который необходимо инжектировать спин, и однородно намагниченной среды, используемой в качестве поляризатора свободных носителей заряда по спину), разделенных проводящей или туннельной прослойкой [1-5]. Такой способ используется, например, в туннельных магнитных контактах с целью считывания информации либо перемагничивания их током [6-9]. В этом случае неравновесный спин инжектируется из одного однородно намагниченного ферромагнетика в другой. В результате такой спин-зависимой инжекции носителей заряда сопротивление оказывается зависящим от взаимной ориентации намагниченностей (ферромагнитное либо антиферромагнитное состояние). При достаточно большой плотности электрического тока инжектируемый спин увлекает за собой намагниченность ферромагнетика, что приводит к перемагничиванию ферромагнитного слоя.

Другой возможный способ заключается в явлении спинового насоса, которое состоит в возникновении потока спина из ферромагнетика в граничащий с ним металл при возбуждении в магнитном материале ферромагнитного резонанса (ФМР). Данный эффект был впервые предложен в 2002 г. [10,11] и с тех пор довольно много исследовался как теоретически, так и экспериментально [12]. В первых работах было показано, что этот эффект приводит к уширению линии поглощения СВЧ-излучения ферромагнетиком при ферромагнитном резонансе (ФМР). Позже было показано, что в среде, в которую инжектируется спин, возникает разность потенциалов в направлении, перпендикулярном равновесному вектору намагниченности источника спина и нормали к границе среды. Механизмами этого явления могут быть обратный спиновый эффект Холла [13] либо эффект Рашбы-Эдельштейна [14]. Указанный транспортный эффект, связанный с инжекцией неравновесного спина в проводник, может быть использован для регистрации ферромагнитного резонанса электрическим методом [15], а также для определения угла спинового эффекта Холла для разных проводников [16]. Эффект спинового насоса возникает как в системе металл/ферромагнитный проводник, так и в системе металл/ферромагнитный диэлектрик [10,17]. В качестве среды, в которую инжектируется спин, как правило выступает немагнитный металл либо полупроводник или магнитный металл, намагниченность которого не играет ключевой роли в механизме возникновения эффекта.

В настоящей работе теоретически рассмотрен ряд явлений, которые могут возникать при инжекции неравновесного спина описанными выше двумя способами в ферромагнетик с неколлинеарным геликоидальным пространственным распределением намагниченности

$$
\mathbf{M}_{h}(\mathbf{r})=(\cos q z, \sin q z, 0),
$$

где $q=\frac{2 \pi}{L_{h}}-$ величина, обратная шагу спирали $L_{h}$. Интерес представляет как определение геометрии системы, 
при которой возможна инжекция заряда в определенную спиновую подзону ферромагнетика, так и обнаружение новых эффектов, связанных с инжекцией в неколлинеарный ферромагнетик неравновесного спина. Наиболее интересным является случай, когда ось геликоида лежит в плоскости границы раздела двух ферромагнетиков и параллельна намагниченности источника спина. В этом случае при спиновом пампинге возникает разность потенциалов в направлении оси геликоида, имеющая чисто обменную природу. При инжекции спина током возникает явление, аналогичное топологическому эффекту Холла [18-21].

\section{2. Теоретическое описание электронов проводимости в геликоидальном ферромагнетике}

Гамильтониан электронов в ферромагнетике с геликоидальным распределением намагниченности записывается в рамках $s-d$ модели Вонсовского [22] и имеет вид

$$
\hat{H}_{h}=-\frac{\hbar^{2}}{2 m_{e}} \Delta+J_{h}\left(\hat{\boldsymbol{\sigma}} \mathbf{M}_{h}\right)
$$

где намагниченность $\mathbf{M}_{h}$ определяется формулой (1), $J_{h}$ - обменная константа в среде с геликоидальной намагниченностью. Как известно из литературы [23-25], волновые функции и спектр электронов проводимости могут быть найдены для гамильтониана (2) точно. Они имеют вид

$$
\begin{aligned}
& \psi_{+}=\exp \left(-i \frac{\varepsilon_{+}}{\hbar} t+i \mathbf{k r}\right)\left(\begin{array}{c}
\delta e^{-i \frac{q}{2} z} \\
e^{i \frac{q}{2} z}
\end{array}\right), \\
& \psi_{-}=\exp \left(-i \frac{\varepsilon_{-}}{\hbar} t+i \mathbf{k r}\right)\left(\begin{array}{c}
e^{-i \frac{q}{2} z} \\
-\delta e^{i \frac{q}{2} z}
\end{array}\right), \\
& \varepsilon_{ \pm}=\frac{\hbar^{2}}{2 m_{e}}\left(\mathbf{k}^{2}+\frac{q^{2}}{4} \pm \sqrt{j_{h}^{2}+k^{2} q^{2}}\right),
\end{aligned}
$$

где $k-z$-компонента волнового вектора, $j_{h}=\frac{2 m_{e}}{\hbar^{2}} J_{h}$ имеет размерность квадрата волнового числа, коэффициент $\delta$, определяющий смешивание компонент спинора, определяется выражением

$$
\delta=\frac{j_{h}}{k q+\sqrt{j_{h}^{2}+k^{2} q^{2}}} \equiv \frac{-k q+\sqrt{j_{h}^{2}+k^{2} q^{2}}}{j_{h}} .
$$

Данное решение часто разлагают в ряд, вводя тот или иной малый параметр. Часто для электронов проводимости считают малым параметр адиабатичности $\beta=\frac{q k_{f}}{J_{h}}$ $\left(k_{f}=\sqrt{\frac{2 m_{e}}{\hbar^{2}} \varepsilon_{f}}-\right.$ импульс Ферми, $\varepsilon_{f}-$ энергия Ферми), равный отношению времени осцилляции спина электрона в обменном поле к времени пролета электроном неоднородности намагниченности $[21,26]$. Однако, в рамках данной работы удобнее пользоваться противоположным пределом, который фактически является учетом обменного взаимодействия в геликоидальной структуре по теории возмущений. Это приближение может быть верно для таких веществ, как гольмий, в котором реализуется геликоидальное распределение намагниченности с параметром адиабатичности порядка нескольких единиц $(\beta \sim 5-10)$ [27]. Параметр $\delta$ и спектр электронов в этом случае можно записать в виде

$$
\begin{gathered}
\delta \approx \frac{j_{h}}{2 k q} \ll 1, \\
\varepsilon_{ \pm}=\frac{\hbar^{2}}{2 m_{e}}\left(\mathbf{k}^{2}+\frac{q^{2}}{4} \pm\left(k q+\frac{j_{h}^{2}}{2 k q}\right)\right) .
\end{gathered}
$$

Как видно из (3), (4), (7), волновые функции в данном приближении близки к волновым функциям свободных электронов в случае, когда ось квантования спина параллельна оси $z$. Важно отметить, что данное разложение справедливо вдали от $k=0$ (при $\left.k \gg j_{h} / q\right)$. В дальнейшем в области малых $k$ приходится учитывать точное решение $(3)-(5)$ для того, чтобы избавиться от сингулярности.

\section{3. Явление спинового насоса В геликоидальный ферромагнетик}

При спиновом пампинге из области с осциллирующей намагниченностью в соседний проводник возникает спиновый ток, который определяется выражением

$$
J_{S_{i j}}=A\left[\mathbf{m} \frac{d \mathbf{m}}{d t}\right]_{j} n_{j},
$$

где выписано только слагаемое, дающее ненулевую среднюю по периоду величину, $\mathbf{n}$ - вектор нормали к границе раздела сред, m - осциллирующая часть намагниченности. Наличие такого неравновесного спина может привести к появлению электрического тока в случае, если область, в которую он инжектирован, намагничена неколлинеарно и в ней пространственные и спиновые степени свободы электронов проводимости связаны. Из соображений симметрии такой ток может быть записан в виде

$$
j_{i} \sim\left(\left[\mathbf{M}_{h} \frac{d \mathbf{M}_{h}}{d x_{i}}\right]\left[\mathbf{m} \frac{d \mathbf{m}}{d t}\right]\right),
$$

где в качестве пространственно-неоднородной намагниченности записана намагниченность геликоида. Ясно поэтому, что для получения (10) необходимо в расчете учитывать слагаемые до второго порядка по обменным константам геликоида и ферромагнетика с осциллирующей намагниченностью. 


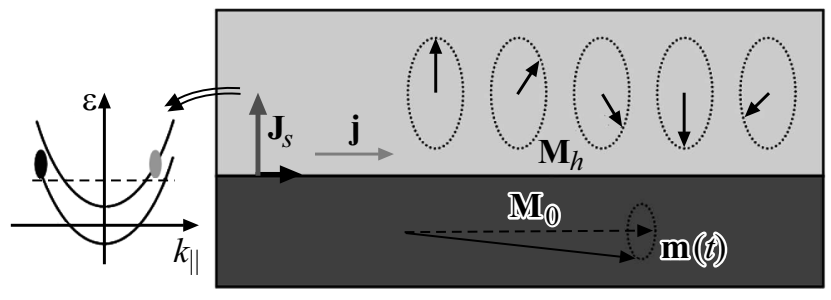

Pис. 1. Возникновение электрического тока при инжекции спина в геликоидальный ферромагнетик из ферромагнетика с осциллирующей во времени намагниченностью.

Из формулы (10) видно, что этот ток имеет чисто обменную природу: спин инжектируется из ферромагнетика с осциллирующей во времени намагниченностью в пространственно-неоднородный ферромагнетик, где за счет обмена на неоднородной намагниченности имеется связь спина и импульса электрона. Это приводит к возникновению потока спин-поляризованных электронов, т. е. электрического тока (см. рис. 1).

Ток вида (10) может быть вычислен в рамках теории, основанной на решении квантово-механической задачи. Гамильтониан ферромагнетика с осциллирующей намагниченностью может быть записан в виде

$$
\hat{H}=-\frac{\hbar^{2}}{2 m_{e}} \Delta+J_{0}\left(\hat{\boldsymbol{\sigma}}, \mathbf{M}_{0}(t)\right)+U,
$$

где намагниченность

$$
\mathbf{M}_{0}(t)=\left(m \cos \Omega t, m \sin \Omega t, \sqrt{1-m^{2}}\right),
$$

$J_{0}$ - обменная константа ферромагнетика с осциллирующей намагниченностью, $\Omega$ - частота осцилляций намагниченности, $U-$ потенциал ферромагнетика с осциллирующей намагниченностью относительно геликоидального ферромагнетика. Мы будем считать, что спиновый пампинг происходит из ферромагнитного изолятора $c U \gg \varepsilon_{f}\left(\varepsilon_{f}-\right.$ энергия Ферми геликоидального ферромагнетика). Решение нестационарного уравнения Шредингера в среде с гамильтонианом (11), а также, расчет явления спинового насоса, подробно описаны в [28]. Следуя этой работе, для вычисления электрического тока необходимо найти отраженные волновые функции для падающих на границу электронов двух спиновых поляризаций, а затем просуммировать их и усреднить по всем квазиимпульсам падающих электронов. Отличие здесь состоит в том, что в то время, как в среде с осциллирующей намагниченностью смешиваются состояния с разной энергией и противоположной проекцией спина на ось $z$, в геликоидально намагниченной среде смешиваются состояния с разным квазиимпульсом вдоль оси геликоида и с противоположной проекцией спина на ось $z$. Это приводит к тому, что в общем случае смешивается бесконечное (счетное) число состояний; данный ряд можно оборвать, если ограничиться вторым порядком по константе обменного взаимодействия в геликоиде. Суммируя затем все вклады в ток от падающего и рассеянного состояний, получим во втором порядке по константам обменного взаимодействия $J_{h}, J_{0}$ и в первом порядке по частоте осцилляций $\Omega$ (учитывая также, что $\left.U \gg \varepsilon_{f}\right)$ :

$$
\mathbf{j}=-\frac{5}{4 \pi^{2}} \frac{e m_{e}}{\hbar^{2}} \frac{k_{\mathrm{F}}}{q} \frac{\Omega J_{f}^{2} J_{h}^{2}}{U^{3}} m^{2} \mathbf{e}_{z},
$$

где $\mathbf{e}_{z}$ - единичный вектор, направленный вдоль оси $z$. Видно, что данный вклад в ток параллелен оси геликоида. При получении (13) не использовалось предположения о малости амплитуды осцилляций намагниченности в источнике спина; однако, полученный ток пропорционален амплитуде осцилляций намагниченности $\mathrm{m}$ во второй степени. Это соответствует феноменологической формуле (10). Кроме того, эффект обратно пропорционален третьей степени потенциала диэлектрика $U$. Эта зависимость соответствует зависимости от данного потенциала инжектируемого при спиновом пампинге из диэлектрика в металл постоянного спина [28].

\section{4. Инжекция спина в геликоидальный ферромагнетик током}

В данном разделе рассматриваются две геометрии. В первом случае ось геликоида направлена вдоль нормали к границе раздела сред с геликоидальным и однородным магнитными моментами. Во втором случае ось лежит в плоскости раздела сред и параллельна намагниченности однородно намагниченной подсистемы. Даны оценки предложенных эффектов; детальное микроскопическое рассмотрение данных задач является предметом будущих исследований.

\section{1. Ось геликоида перпендикулярна границе раздела сред}

В данной геометрии возможна инжекция спина в определенную спиновую подзону геликоидального ферромагнетика при правильном выборе направления намагниченности источника спина $\mathbf{M}_{0}$ (если считать, что инжектируемые электроны полностью поляризованы по спину).

Это направление нетрудно определить из решения простой квантово-механической задачи. Пусть на плоскую границу раздела сред из однородного ферромагнетика падает электрон, поляризованный вдоль вектора намагниченности $\tilde{\psi}=\left(\begin{array}{l}1 \\ 0\end{array}\right)$, при этм ось квантования спина параллельна направлению намагниченности в ферромагнетике $\mathbf{M}_{0}$. Само же направление вектора намагниченности определяется из условия, что такой электрон при прохождении через границу порождает не суперпозицию состояний, а одно состояние (3) либо (4) (см. рис. 2). Записывая граничные условия непрерывности волновой функции и ее производной, нетрудно определить требуемое направление намагниченности $\mathbf{M}_{0}$. В приближении, 

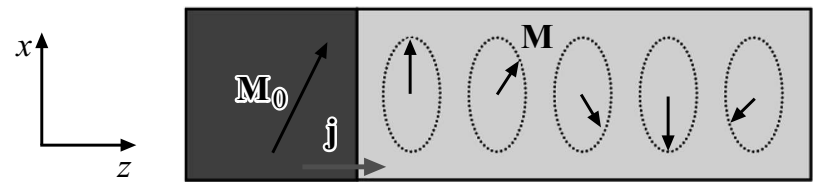

Pис. 2. Инжекция спина током в определенную подзону геликоидального ферромагнетика. Ось геликоида перпендикулярна границе раздела сред.

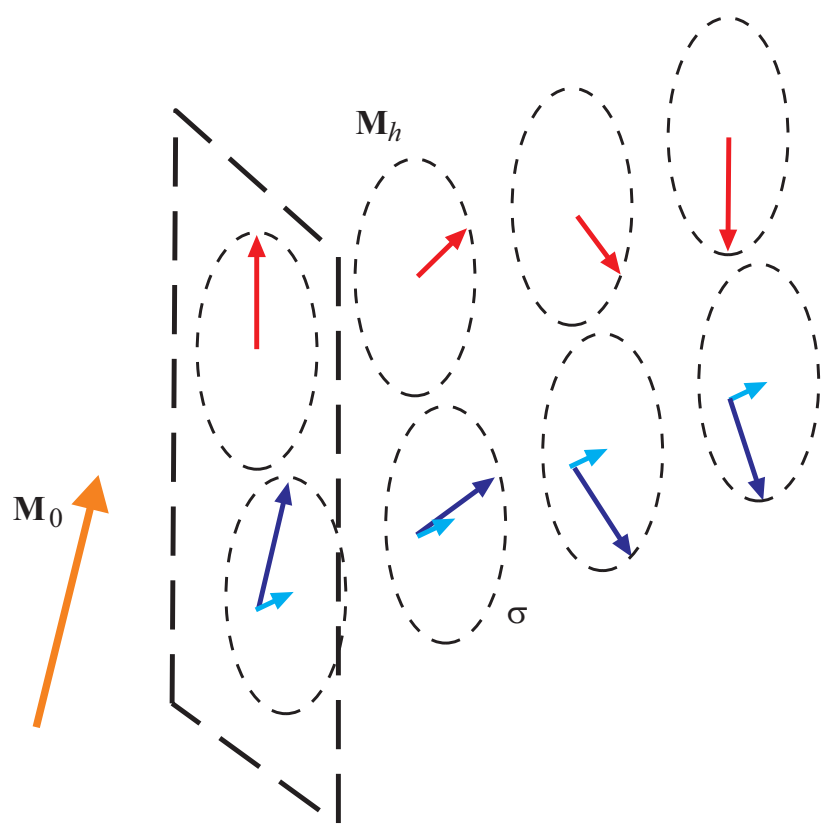

Рис. 3. Намагниченность $\mathbf{M}_{h}$ и спин $\sigma$ электронов проводимости в геликоидальном ферромагнетике.

когда энергия электрона $\varepsilon \gg J_{h}, J_{0}, \frac{\hbar^{2} q^{2}}{2 m_{e}}$, оно может быть записано в виде

$$
\mathbf{M}_{0 \pm}= \pm\left(\frac{2 \delta}{1+\delta^{2}} \mathbf{e}_{x}+\frac{\delta^{2}-1}{\delta^{2}+1} \mathbf{e}_{z}\right)
$$

для волновых функций (3) и (4) соответственно, где система координат выбрана в соответствии с рис. 2 (ось $z$ параллельна нормали к границе раздела сред и оси геликоида, ось $x$ параллельна намагниченности геликоида на границе). Заметим, что намагниченность $\mathbf{M}_{0}$, необходимая для инжекции спина в определенную подзону геликоидального ферромагнетика, не точно параллельна намагниченности ферромагнетика на границе. Это связано с тем, что электроны в геликоидальном ферромагнетике имеют спин не точно параллельный локальной намагниченности, а приобретают неадиабатический спин, направленный вдоль оси геликоида $z$. Направление оказывается точно параллельным направлению спина электрона соответствующей спиновой подзоны на границе геликоида (см. рис. 3). Если взять для оценки параметры $J_{h}=0.1 \mathrm{eV}, L_{h}=\frac{2 \pi}{q}=30 \mathrm{~nm}, k_{f}=5.4 \cdot 10^{7} \mathrm{~cm}^{-1}$, угол отклонения $\mathbf{M}_{0}$ от оси $x$, необходимый для инжекции спина в одну из подзон геликоидального ферромагнетика при $k_{z} \sim k_{f}$ составляет порядка $18^{\circ}$, что является весьма значительной величиной.

В реальном ферромагнетике электроны поляризованы по спину не стопроцентно. При этом электроны противоположных спиновых поляризаций инжектируются в противоположные спиновые подзоны в геликоиде. Тем не менее, поскольку одна из спиновых поляризаций доминирует над другой (разность числа электронов пропорциональна $\frac{J_{0}}{\varepsilon_{f}}$ ), указанным в настоящей работе выбором геометрии системы можно организовать инжекцию в требуемую спиновую подзону магнитного геликоида большего числа электронов, чем в противоположную (соответственно, разность числа электронов здесь также будет пропорциональна $\left.\frac{J_{0}}{\varepsilon_{f}}\right)$.

\section{2. Ось геликоида параллельна границе раздела сред}

Поскольку в данном случае спин электронов в геликоиде меняется на границе раздела сред в направлении оси геликоида, найти направление намагниченности $\mathbf{M}_{0}$, при котором осуществляется инжекция спина только в одну спиновую подзону (при стопроцентной поляризации инжектируемых электронов по спину), не получается. (Математически это обусловлено тем, что на границе приходится „сшивать“ волновые функции с разными проекциями волнового вектора на границу раздела сред в спиноре.) С другой стороны, в данной геометрии возможен интересный транспортный эффект, который аналогичен топологическому эффекту Холла [18-21]. С точки зрения симметрии его можно понять следующим образом. Из однородно намагниченного ферромагнетика в неоднородный магнетик инжектируются электроны, поляризованные по спину вдоль $\mathbf{M}_{0}$. За счет обменного взаимодействия в неоднородном ферромагнетике их спиновые степени свободы оказываются связанными с пространственными. В результате этого возникает электрический ток вдоль оси геликоида

$$
j_{H i} \sim\left(\left[\mathbf{M}_{h} \frac{d \mathbf{M}_{h}}{d x_{i}}\right] \mathbf{M}_{0}\right),
$$

который направлен перпендикулярно протекающему из одного ферромагнетика в другой току и, таким образом, является холловским (см. рис. 4). Для его существования необходима, как и в случае топологического эффекта Холла, некомпланарность трех векторов, присутствующих в формуле (15).

Для демонстрации механизма возникновения данного эффекта была решена простая задача об инжекции электрона, имеющего поляризацию вдоль оси геликоида $z$, которая параллельна границе раздела двух сред

$$
\psi_{0+} \sim \exp \left(i k_{x} x+i\left(k-\frac{q}{2}\right) z\right)\left(\begin{array}{l}
1 \\
0
\end{array}\right),
$$

где ось $x$ параллельна нормали к границе раздела сред, ось $z$ лежит вдоль оси геликоида; поперечная 


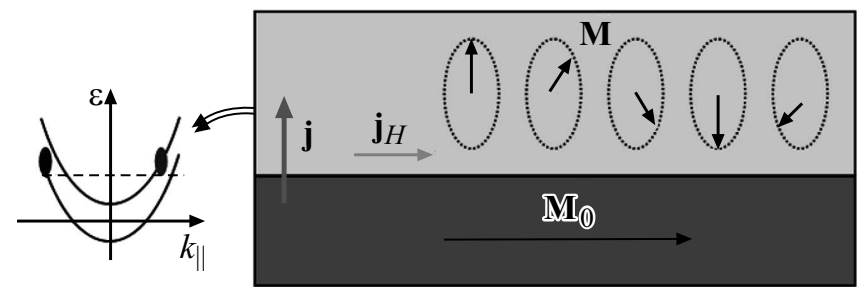

Рис. 4. Возникновение эффекта Холла при инжекции в геликоидальный ферромагнетик электрического тока, поляризованного по спину вдоль оси геликоида. Ось геликоида параллельна границе раздела сред.

компонента квазиимпульса и зависящий от времени множитель опущены. Волновая функция (16) порождает в геликоиде волновые функции электронов двух спиновых поляризаций с разным весом. Записав простые граничные условия непрерывности волновой функции и ее производной вдоль нормали к границе, нетрудно найти данный вес. Аналогичным образом нужно учесть инжекцию электронов с противоположным спином

$$
\psi_{0-} \sim \exp \left(i k_{x} x+i\left(k+\frac{q}{2}\right) z\right)\left(\begin{array}{l}
0 \\
1
\end{array}\right) .
$$

Необходимо затем просуммировать токи, порождаемые всеми такими решениями, по спину квазиимпульсу. Для удобства мы выбрали квазиимпульс вдоль оси $z$ сдвинутым на $\pm \frac{q}{2}$ для инжекции двух разных спиновых поляризаций. Это соответствующим образом отражается на пределах интегрирования.

Ток, возникающий в результате инжекции спина (16), равен

$$
j_{+}=-\frac{e \hbar}{2 m_{e}}\left(k-\frac{q}{2}\left(\frac{\delta^{2}-1}{\delta^{2}+1}\right)^{2}\right),
$$

а при инжекции спина (17), соответственно

$$
j_{-}=-\frac{e \hbar}{2 m_{e}}\left(k+\frac{q}{2}\left(\frac{\delta^{2}-1}{\delta^{2}+1}\right)^{2}\right) .
$$

Если просуммировать ток (18) для $k$ и $-k$, он оказывается в точности равным току (19), аналогично просуммированному по $k$ и $-k$. Поэтому при таком выборе квазиимпульса вдоль оси $z$ вклад дают только асимметричные пределы, связанные со сдвигом квазиимпульса на $\pm \frac{q}{2}$, а также различие пределов для двух спиновых подзон ферромагнетика. Поскольку ненулевой вклад возникает в результате интегрирования вблизи пределов, можно оценить интеграл, считая обменную константу $J_{h}$ малой по сравнению с энергией Ферми $\varepsilon_{f}$. Оценивая также интеграл по другим компонентам квазиимпульса как $\frac{2 m_{e}}{\hbar^{2}} \mathrm{eV}$, где $V$ - приложенное к системе напряжение, получим оценку для тока

$$
\mathbf{j}_{H}=-e^{2} \frac{\left(2 m_{e}\right)^{3 / 2}}{\hbar^{4}} \frac{J_{h}^{2} J_{0} V}{q \varepsilon_{f}^{3 / 2}} \mathbf{e}_{z}
$$

Данная оценка соответствует феноменологической формуле (15). Стоит отметить, что в отличие от общепринятого топологического эффекта Холла в некомпланарных ферромагнетиках, который пропорционален масштабу неоднородности намагниченности в третьей степени, здесь входит только первая производная намагниченности в неколлинеарном геликоидальном магнетике; два порядка по масштабу неоднородности здесь пропадают из-за наличия резкой границы двух ферромагнетиков с разной ориентацией и структурой намагниченности. Поэтому попытка экспериментального наблюдения эффекта, аналогичного топологическому эффекту Холла, в такой системе может представлять интерес.

\section{5. Заключение}

В настоящей работе исследованы эффекты, возникающие при инжекции неравновесного спина в геликоидальный ферромагнетик. Продемонстрировано, что при спиновом пампинге в случае, когда ось геликоида параллельна границе с источником спина и его равновесной намагниченности, возникает дополнительный механизм конверсии инжектируемого спинового тока в электрический за счет обенного взаимодействия в неколлинеарном ферромагнетике. Такой ток, в отличие от традиционного эффекта конверсии спинового тока в электрический за счет обратного спинового эффекта Холла, течет вдоль оси геликоида и, соответственно, вдоль направления равновесной намагниченности ферромагнетика, из которого инжектируется спин. При инжекции спина электрическим током для случая, когда ось геликоида перпендикулярна границе, через которую происходит инжекция, определены условия, при которых происходит заполнение электронами заданной спиновой подзоны. Для случая, когда ось геликоида параллельна границе раздела, продемонстрировано возникновение эффекта, аналогичного топологическому эффекту Холла.

\section{Финансирование работы}

Работа выполнена при поддержке РНФ, грант № 19-72-00130.

\section{Конфоликт интересов}

Авторы заявляют, что у них нет конфликта интересов.

\section{Список литературы}

[1] M.N. Baibich, J.M. Broto, A. Fert, F. Nguyen Van Dau, F. Petroff, P. Etienne, G. Creuzet, A. Friederich, J. Chazelas. Phys. Rev. Lett. 61, 2472 (1988).

[2] J.C. Slonczewski. J. Magn. Magn. Mater. 159, L1 (1996).

[3] L. Berger. Phys. Rev. B 54, 9353 (1996).

[4] J.S. Moodera, L.R. Kinder, T.M. Wong, R. Meservey. Phys. Rev. Lett. 74, 16, 3273 (1995). 
[5] I. Zutic, J. Fabian, S.D. Sarma. Rev. Mod. Phys. 76, 323 (2004).

[6] K. Ohgushi, S. Murakami, N. Nagaosa. Phys. Rev. B 62, R6065 (1999).

[7] J.A. Katine, E.E. Fullerton. J. Magn. Magn. Mater. 320, 1217 (2008).

[8] S.S.P. Parkin, M. Hayashi, L. Thomas. Science 320, 190 (2008).

[9] D. Atkinson, D.S. Eastwood, L.K. Bogart. Appl. Phys. Lett. 92, 022510 (2008).

[10] Y. Tserkovnyak, A. Brataas, G.E.W. Bauer. Phys. Rev. Lett. 88, 117601 (2002).

[11] A. Brataas, Y. Tserkovnyak, G.E.W. Bauer, B.I. Halperin. Phys. Rev. B 66, 060404(R) (2002).

[12] Y. Tserkovnyak, A. Brataas, G.E.W. Bauer, B.I. Halperin. Rev. Mod. Phys. 77, 1375 (2005).

[13] J. Sinova, S.O. Valenzuela, J. Wunderlich, C.H. Back, T. Jungwirth. Rev. Mod. Phys. 87, 1213 (2015).

[14] J.C. Rojas Sanchez, L. Vila, G. Desfonds, S. Gambarelli, J.P. Attané, J.M. De Teresa, C. Magén, A. Fert. Nature Communications 4, 2944 (2013).

[15] E. Saitoh, M. Ueda, H. Miyajima, G. Tatara. Appl. Phys. Lett. 88, 182509 (2006).

[16] H. Wang, C. Du, P.C. Hammel, F. Yang. Appl. Phys. Lett. 104, 202405 (2014).

[17] G. Tatara, S. Mizukami. Phys. Rev. B 96, 064423 (2017).

[18] Y. Taguchi, Y. Oohara, H. Yoshizawa, N. Nagaosa, Y. Tokura. Science 291, 2573 (2001).

[19] A. Neubauer, C. Pfleiderer, B. Binz, A. Rosch, R. Ritz, P.G. Niklowitz, P. Böni. Phys. Rev. Lett. 102, 186602 (2009).

[20] S. Onoda, N. Nagaosa. Phys. Rev. Lett. 90, 19, 196602-1 (2003).

[21] Y. Aharonov, A. Stern. Phys. Rev. Lett. 69, 25, 3593 (1992).

[22] С.В. Вонсовский. Магнетизм. Наука, Москва (1971).

[23] В.М. Матвеев, Э.Л. Нагаев. ЖЭТФ 69, 2151 (1975).

[24] Э.Л. Нагаев. Физика магнитных полупроводников. Наука, M. (1979).

[25] M. Calvo. Phys. Rev. B 19, 5507 (1978).

[26] F. Zhou. Phys. Rev. B 70, 125321 (2004).

[27] W.C. Koehler. J. Appl. Phys. 36, 3, 1078 (1965).

[28] Е.А. Караштин. ФТТ 61, 9, 1634 (2019).

Редактор Ю.Э. Китаев 